\title{
Drug-eluting beads bronchial arterial chemoembolization plus intercostals arterial infusion chemotherapy is effective and well- tolerated in treating non-small cell lung cancer patients with refractory malignant pleural effusion
}

\author{
Xiaofei Liu ${ }^{1 \#}$, Hu Lin ${ }^{2 \#}$, Qin Wang ${ }^{3 \#}$, Mi Mu², Pan Pan $^{2}$, Fangfang Tian ${ }^{2}$, Rui Zhang', Weiguo Zhao', \\ Pengtao $\mathrm{Bao}^{2,3}$ \\ ${ }^{1}$ Department of Nuclear Medicine, The Eighth Medical Center of PLA General Hospital, Beijing, China; ${ }^{2}$ Department of Pulmonary and Critical \\ Care Medicine, The Eighth Medical Center of Chinese PLA General Hospital, College of Pulmonary and Critical Care Medicine, Chinese PLA \\ General Hospital, Beijing, China; ${ }^{3}$ Graduate School, Hebei North University, Zhangjiakou, China \\ Contributions: (I) Conception and design: X Liu, H Lin, Q Wang; (II) Administrative support: W Zhao, P Bao; (III) Provision of study materials \\ or patients: All authors; (IV) Collection and assembly of data: X Liu, H Lin, Q Wang, M Mu, P Pan, F Tian, R Zhang; (V) Data analysis and \\ interpretation: W Zhao, P Bao; (VI) Manuscript writing: All authors; (VII) Final approval of manuscript: All authors. \\ \#These authors contributed equally to this work. \\ Correspondence to: Pengtao Bao. Department of Pulmonary and Critical Care Medicine, The Eighth Medical Center of Chinese PLA General \\ Hospital, College of Pulmonary and Critical Care Medicine, Chinese PLA General Hospital, No. 17, Heishanhu Road, Beijing 100093, \\ China; Graduate School, Hebei North University, No.11 South Diamond Road, High-tech District, Zhangjiakou 075000, China. Email: \\ baikangshao9706@163.com; Weiguo Zhao. Department of Pulmonary and Critical Care Medicine, The Eighth Medical Center of Chinese PLA \\ General Hospital, College of Pulmonary and Critical Care Medicine, Chinese PLA General Hospital, No. 17, Heishanhu Road, Beijing 100093, \\ China. Email: weikesi998@126.com.
}

Background: The study aimed to explore the efficacy and safety of drug-eluting beads bronchial arterial chemoembolization (DEB-BACE) plus intercostals arterial infusion chemotherapy in non-small cell lung cancer (NSCLC) patients with refractory malignant pleural effusion (MPE).

Methods: 17 NSCLC patients with refractory MPE treated by DEB-BACE plus the intercostals arterial infusion chemotherapy (DEB-BACE group) were recruited. Their treatment response [complete remission (CR), partial remission (PR), overall efficacy, failure] for MPE was assessed at 1 month after therapy; adverse effects were recorded; MPE progression-free survival and overall survival (OS) were calculated. Moreover, 19 NSCLC patients with refractory MPE treated by conventional chemotherapy were reviewed as control (chemotherapy group), then their medical records were collected.

Results: With respect to MPE response, DEB-BACE group exhibited increased CR (82.4\% vs. $10.5 \%$, $\mathrm{P}<0.001)$ and overall efficacy (100.0\% vs. 52.6\%, $\mathrm{P}=0.001)$, similar PR (17.6\% vs. $42.1 \%, \mathrm{P}=0.112)$ while less failure $(0.0 \%$ vs. $47.4 \%, \mathrm{P}=0.001)$ compared to chemotherapy group. Furthermore, OS was prolonged in DEB-BACE group (median: 13.4; 95\% CI: 11.0-15.8 months) than chemotherapy group (median: 7.0; $95 \%$ CI: 4.4-9.6 months) $(\mathrm{P}=0.002)$. Further analyses displayed that in DEB-BACE group, CR was associated with improved ECOG score and longer MPE progression-free survival, and adverse events mainly included fever, chest distress/pain, gastrointestinal side effects, myelosuppression, rash and hemoptysis, which were all mild and tolerable.

Conclusions: DEB-BACE plus intercostals arterial infusion chemotherapy could serve as a salvage treatment option for NSCLC patients with refractory MPE.

Keywords: Non-small cell lung cancer (NSCLC); refractory malignant pleural effusion (MPE); drug-eluting beads bronchial arterial chemoembolization (DEB-BACE); intercostals arterial infusion chemotherapy; efficacy; safety 
Submitted Apr 07, 2020. Accepted for publication Aug 13, 2020.

doi: $10.21037 /$ jtd-20-1603

View this article at: http://dx.doi.org/10.21037/jtd-20-1603

\section{Introduction}

Malignant pleural effusion (MPE) is an exudative effusion with malignant cells, which is a common symptom and accompanying manifestation of metastatic disease arising from either primary mesotheliomas or secondary malignancies $(1,2)$. It affects up to $15 \%$ of all cancer patients and is considerably common in non-small cell lung cancer (NSCLC), which seriously impairs the quality of life and correlates with a significant reduction in life expectancy of NSCLC patients (3). For the majority of NSCLC patients with MPE, it is required to receive a rapid intervention (such as thoracentesis, pleurodesis and indwelling pleural catheters) to relieve symptoms related to fluid accumulation $(4,5)$. Whereas the frequent complications and high recurrence rate after these interventions limit their clinical benefits, and part of the patients may present with poor responses after treated by these common interventions (categorized as refractory MPE), thus the management of refractory MPE in NSCLC patients is still far from satisfaction $(5,6)$.

The common surgical interventions for relieving MPE symptoms provide a persistent communication through which the fluid could be drained, meanwhile, other than these interventions, there are some other therapeutic opinions focus on the regional delivery of drugs for lung cancer therapy, aiming to eventually repress the exudative effusion of malignant cells to the pleural space (4). Drugeluting beads transarterial chemoembolization (DEBTACE) is a minimally invasive technique consisting of injection of antitumor drugs and embolization in tumorfeeding arteries (7). With the strong antineoplastic effect and ability of inducing ischemic necrosis of the targeted tumor, it has been used successfully to treat liver cancers for many years (8). Similar to DEB-TACE, DEB-bronchial arterial chemoembolization (BACE) is initiated on the basis that bronchial artery is the main feeding artery for lung tumors, thus DEB-BACE may improve the curative effect with the increased local drug concentration and milder systemic cytotoxicity in NSCLC patients with refractory MPE $(9,10)$.

With regard to the application of DEB-BACE in lung cancer patients, the related evidence is limited: two previous studies display that DEB-BACE decreases invasion to the right main bronchus and improves hemoptysis in advanced lung cancer patients; and another one shows the good survival benefit from DEB-BACE with well-tolerance in NSCLC patients (10-12). Besides, the intercostals arterial infusion chemotherapy, which is another intra-arterial intervention able to deliver a high local concentration of the anti-cancer drug to the tumor tissue, is often used combining with other treatments and has displayed certain achievements of treatment response in lung cancers $(13,14)$. These data reveal the acceptable efficacy and toleration of DEB-BACE and the intercostals arterial infusion chemotherapy in NSCLC patients, whereas their efficacy for treating refractory MPE in NSCLC patients is unknown. Hence, we proposed a salvage therapy consisting of DEB-BACE plus intercostals arterial infusion chemotherapy, and aimed to explore its efficacy and safety in treating NSCLC patients with refractory MPE.

\section{Methods}

\section{Ethical approval}

The study was approved by the Institutional Review Board of The Eighth Medical Center of PLA General Hospital. Written informed consents were obtained from all patients or their families. The study was conducted in accordance with the Declaration of Helsinki (as revised in 2013). All patients who were prospectively recruited in our study provided written informed consent themselves, while for the patients in control cohort who were retrospectively enrolled but had died, so we had to obtain the written consents from their families. Additionally, this study was registered on Chinese Clinical Trial Registry (http://www.chictr.org.cn), with the registry number of ChiCTR2000033063.

\section{DEB-BACE cohort}

Seventeen NSCLC patients with refractory MPE who treated by DEB-BACE plus the intercostals arterial infusion chemotherapy in our hospital between January 2017 and March 2019 were recruited in this study, and they were categorized into DEB-BACE group. The inclusion criteria 
were: (I) histopathologically diagnosed as NSCLC including lung adenocarcinoma and squamous cell carcinoma; (II) age 18-75 years, regardless of gender; (III) refractory MPE, which was defined as poorly controlled pleural effusion after pleurodesis or pleural drainage, and no obvious improvement after 2 or more times of chemotherapy; (IV) Eastern Cooperative Oncology Group (ECOG) score of physical fitness $\leq 2$ points; (V) the life expectancy more than 6 months. The exclusion criteria were: (I) complicated with extensive and uncontrolled extrapulmonary metastases; (II) suitable for targeted drug therapy confirmed by gene mutation detection; (III) history of other malignant tumors, or currently complicated with other malignant tumors (except for cured basal or squamous cell skin cancer or cervical carcinoma in situ); (IV) white blood cells $<3 \times 10^{9} / \mathrm{L}$, platelet count $<50 \times 10^{9} / \mathrm{L}$, and hemoglobin $<90 \mathrm{~g} / \mathrm{L}$; (V) liver and kidney dysfunction (creatinine $>2 \mathrm{mg} / \mathrm{L}$; aspartate aminotransferase (AST) and/or alanine aminotransferase $($ ALT) $>$ twice of the normal upper limit); (VI) coagulation dysfunction (international normalized ratio (INR) $>1.5$ ) or a known bleeding disorder, or ongoing anticoagulant therapy; (VII) patients with active infection requiring antibiotic treatment; (VIII) hypersensitivity to contrast agent; (IX) pregnant or lactating women.

\section{Data collection}

Baseline data for patients in the DEB-BACE group were documented, including demographic features (age, gender, history of smoke), ECOG score, and histological type, TNM stage, and previous chemotherapy times.

\section{Treatment}

For 17 patients in DEB-BACE group, they were treated by CalliSpheres ${ }^{\circledR}$ DEB-BACE plus the intercostals arterial infusion chemotherapy, as follows:

(I) Intercostals arterial infusion chemotherapy: microcatheter was superselectively inserted into the intercostal artery of the affected side, and $40 \%$ of chemotherapy drugs were infused into the intercostal artery infusion chemotherapy. The chemotherapy regimens included pemetrexed $\left(375 \mathrm{mg} / \mathrm{m}^{2}\right)$ plus carboplatin $\left(225 \mathrm{mg} / \mathrm{m}^{2}\right)$ for adenocarcinoma and gemcitabine $\left(750 \mathrm{mg} / \mathrm{m}^{2}\right)$ plus carboplatin $\left(225 \mathrm{mg} / \mathrm{m}^{2}\right)$ for squamous cell carcinoma.
(II) CalliSpheres ${ }^{\circledR}$ DEB-BACE procedures: bronchial arteriography was performed to identify the tumor blood supply artery branch, then microcatheter superselective catheterization was carried out. Subsequently, the remaining $60 \%$ of the chemotherapy drugs were infused into the bloodsupply bronchial arterial of tumor. Following that, a bottle of Callispheres ${ }^{\circledR}$ beads (ranging 300-500 microns in diameter) loading epirubicin $\left(1.5 \mathrm{mg} / \mathrm{m}^{2}\right)$ were infused into blood-supply bronchial arterial of tumor at a speed of $1 \mathrm{~mL} / \mathrm{min}$ for chemoembolization. The chemoembolization was suspended when the blood flow in the tumor supply artery was slow and nearly stopped. Fiver min later, angiography was performed again to determine whether partial supplementary embolization should be administered. If a bottle of Callispheres ${ }^{\circledR}$ beads was not enough for complete embolization (due to the abundant blood supply of tumor), the blank Callispheres ${ }^{\circledR}$ beads were added to achieve the complete embolization (the blood flow in the tumor supply artery was stopped), which was conducted according to the actual situation of the patient. A typical case's treatment process was shown in the Figure 1. Briefly, right bronchial artery was thickened and had common stem with intercostal artery [2-3], meanwhile, the contrast media staining for tumor was seen in pulmonary parenchymal phase by arteriography (Figure 1A); the right intercostal artery [4-5, 5-6] was also found to be thickened, then intercostals arterial infusion chemotherapy was performed (Figure 1B); thereafter, the microcatheter was inserted into the right bronchial artery (superselection was performed to avoid the intercostal artery), and epirubicin-loaded Callispheres ${ }^{\circledR}$ beads were infused into blood-supply bronchial arterial (Figure 1C); a month later, compared with pleural effusion status before treatment (Figure 1D. pleural effusion was seen in the right thorax), the pleural effusion was disappeared (Figure 1E).

(III) Sequential therapy: for patients with no remission and a part of patients with partial remission $(\mathrm{PR})$ after first cycle therapy, they were administered with next cycle of treatment. The treatment interval was 1 month, and the treatment was no more than 3 cycles in total. As for patients with 

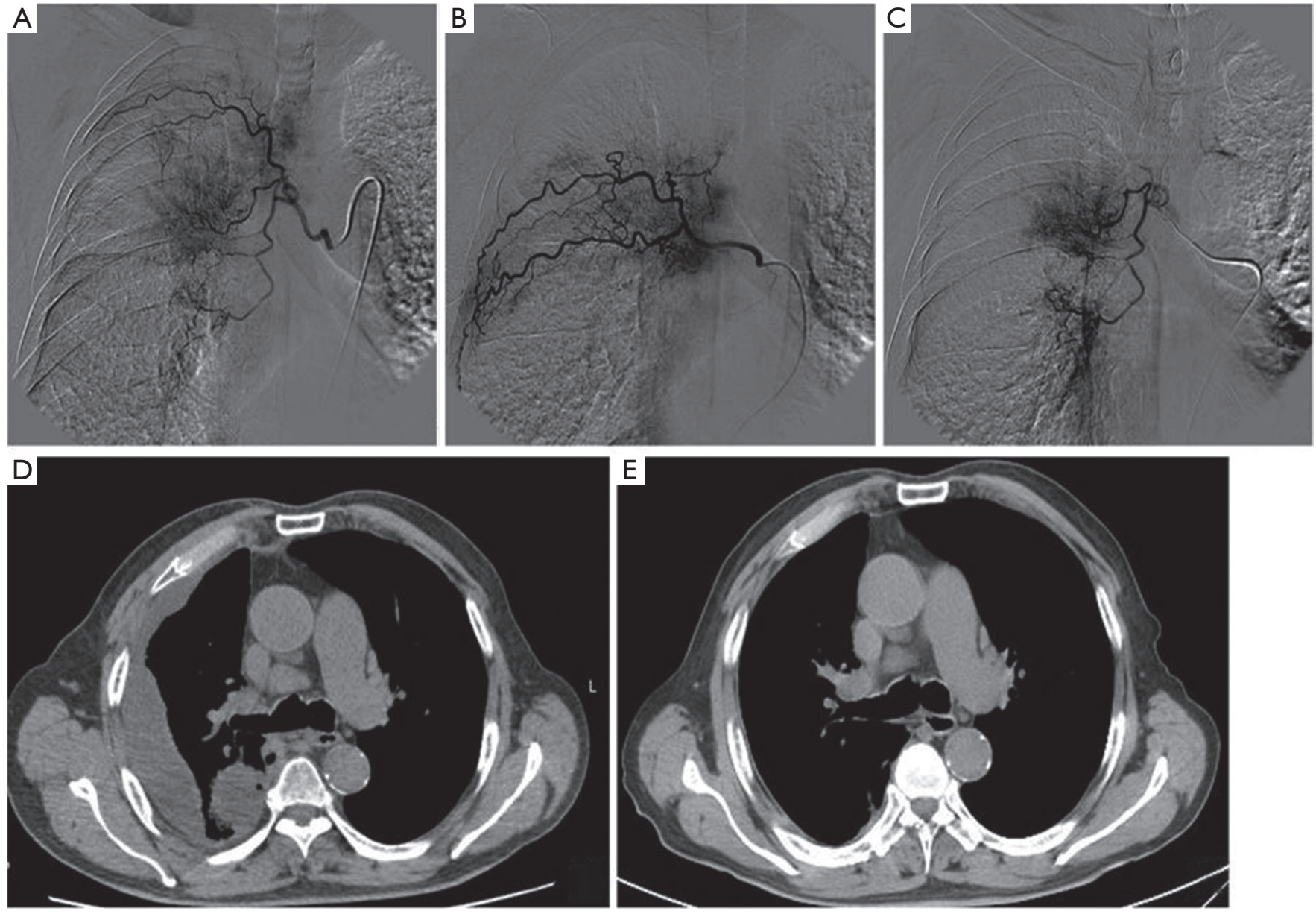

Figure 1 A typical case's treatment process by DEB-BACE. The thickened right bronchial artery and the contrast media staining for tumor by arteriography (A); the thickened right intercostal artery and the performed intercostals arterial infusion chemotherapy shown by arteriography (B); the process of microcatheter inserting into the right bronchial artery and epirubicin-loaded Callispheres ${ }^{\circledR}$ beads being infused into blood-supply bronchial arterial shown by arteriography (C); CT scan for pleural effusion status before treatment (D); CT scan for pleural effusion status a month later after treatment (E). DEB-BACE, drug-eluting beads bronchial arterial chemoembolization; CT, computed tomography.

complete remission (CR) and a part of patients with PR after first cycle therapy, they were treated with systemic chemotherapy of pemetrexed $\left(500 \mathrm{mg} / \mathrm{m}^{2}\right)$ plus carboplatin $\left(300 \mathrm{mg} / \mathrm{m}^{2}\right)$ by intravenous injection, repeated every 21 days (for adenocarcinoma), or treated with gemcitabine $\left(1,000 \mathrm{mg} / \mathrm{m}^{2}\right)$ plus carboplatin $\left(300 \mathrm{mg} / \mathrm{m}^{2}\right)$ by intravenous injection, repeated every 21 days (for squamous cell carcinoma), until the patients withdrew from the study.

(IV) Collaborative therapy: during the study, patients allowed to receive treatment for nutrition, pain, antiemetic, antifebrile, correction of myelosuppression and other symptomatic support treatments. While except for therapies described above, the following collaborative treatments were not allowed: (I) other systemic anti-tumor therapy: intravenous chemotherapy, targeted therapy, immunotherapy, etc.; (II) partial anti-tumor therapy: surgery, radiotherapy (including seed implantation), ablation therapy, etc.; (III) traditional Chinese medicine treatment with anti-tumor effect. 


\section{Evaluation criteria}

Treatment response for MPE was assessed at 1 month after therapy, according to previous studies $(15,16)$. which was defined as follows: CR: after treatment, pleural effusion completely disappeared, and symptoms of chest tightness and shortness of breath were completely relieved. No effusion was observed after $\mathrm{X}$-ray and b-ultrasound examination, which was maintained for more than 4 weeks; PR: the pleural effusion was reduced by more than $50 \%$, and the symptoms were significantly improved, which was maintained for more than 4 weeks; failure: after treatment, the pleural effusion was reduced less than $50 \%$, or the pleural effusion was increased, and the symptoms were not improved, or were aggravated. Overall efficacy for MPE was defined as $\mathrm{CR}+\mathrm{PR}$. Besides, performance status was evaluated according to ECOG score criteria: improved: ECOG score reduced $>1$ for more than 4 weeks; stable: no significant changes in ECOG score; deteriorated: ECOG score increased $>1$ point. Besides, adverse events for 17 patients in the DEB-BACE group were recorded during the followup period, according to the Common Terminology Criteria for Adverse Events (CTCAE) (version 4.0).

\section{Follow-up}

Patients were followed up once a month from the start of treatment, and the follow-up examinations included physical examination, medication history, progress of pleural effusion, blood routine, liver and kidney function, chest imaging or chest ultrasound. All patients were continuously followed up to patients' death. The last follow-up date was December 2019. The MPE progression-free survival was defined as the time interval from initiation of therapy to MPE recurrence, progression or death. The overall survival (OS) was defined as the time interval from initiation of therapy to patients' death.

\section{Control cohort}

In addition, 19 NSCLC patients with refractory MPE treated by conventional chemotherapy in our hospital were reviewed and included as control cohort in the analysis, and they were categorized into chemotherapy group. The inclusion criteria were: (I) histopathologically diagnosed as NSCLC including lung adenocarcinoma and squamous cell carcinoma; (II) age 18-75 years, regardless of gender; (III) refractory MPE, which was defined as poorly controlled pleural effusion after pleurodesis or pleural drainage, and no obvious improvement after 2 or more times of chemotherapy; (IV) ECOG score of physical fitness $\leq 2$ points. The exclusion criteria were: (I) history of DEB-BACE; (II) history of other malignant tumors; (III) clinical data or follow-up data were missing. Their ethical approval/consent for their data included were gained. All of them had matched age and gender with patients in DEBBACE group. The data of these patients were collected from medical records. Following pleurodesis or pleural drainage, due to poor efficacy, they were treated by systemic chemotherapy of pemetrexed $\left(500 \mathrm{mg} / \mathrm{m}^{2}\right)$ plus carboplatin $\left(300 \mathrm{mg} / \mathrm{m}^{2}\right)$ by intravenous injection (for adenocarcinoma), repeated every 21 days, or gemcitabine $\left(1,000 \mathrm{mg} / \mathrm{m}^{2}\right)$ plus carboplatin $\left(300 \mathrm{mg} / \mathrm{m}^{2}\right)$ by intravenous injection (for squamous cell carcinoma), repeated every 21 days.

\section{Statistical analysis}

SPSS 22.0 statistical software (IBM, USA) was used to analysis, and GraphPad Prism (GraphPad Prism lnc., USA) was used for Figure plotting. Data were expressed as mean \pm standard deviation (SD), range, median and $95 \% \mathrm{CI}$, or count (percentage). Differences among groups were analyzed by Student' $t$ test, Chi-square test or Wilcoxon rank sum test. Kaplan-Meier curve was used to display MPE progression-free survival and OS. Comparison of KaplanMeier curve was determined by log-rank test. A P value $<0.05$ was considered statistically significant.

\section{Results}

\section{Characteristics of NSCLC patients with refractory MPE}

The mean age was $60.3 \pm 9.6$ years (range, $42-74$ years) in

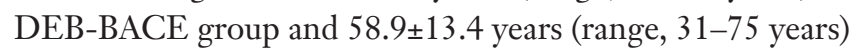
in chemotherapy group (Table 1$)$. No difference of demographic features (age $(\mathrm{P}=0.734)$, gender $(\mathrm{P}=0.738)$ or history of smoke $(\mathrm{P}=0.985))$ was observed between DEBBACE group and chemotherapy group. For ECOG score, 2 (11.8\%), $10(58.8 \%), 5$ (29.4\%) patients in DEB-BACE group and $2(10.5 \%), 12(63.2 \%), 5(26.3 \%)$ patients in chemotherapy group showed ECOG score 0, 1, 2 respectively. As to histological type, $11(64.7 \%)$ patients were with adenocarcinoma and $6(35.3 \%)$ patients were with squamous carcinoma in DEB-BACE group; 11 (57.9\%) patients were with adenocarcinoma and $8(42.1 \%)$ patients were with squamous carcinoma in chemotherapy group. No 
Table 1 Characteristics of patients

\begin{tabular}{|c|c|c|c|}
\hline Items & $\begin{array}{c}\text { DEB-BACE } \\
\text { group }(\mathrm{N}=17)\end{array}$ & $\begin{array}{l}\text { Chemotherapy } \\
\text { group }(\mathrm{N}=19)\end{array}$ & $P$ value \\
\hline Age (years) & & & 0.734 \\
\hline Mean \pm SD & $60.3 \pm 9.6$ & $58.9 \pm 13.4$ & \\
\hline Range & $42-74$ & $31-75$ & \\
\hline Gender, No. (\%) & & & 0.738 \\
\hline Male & 9 (52.9) & $9(47.4)$ & \\
\hline Female & $8(47.1)$ & $10(52.6)$ & \\
\hline History of smoke, No. (\%) & & & 0.985 \\
\hline Yes & $8(47.1)$ & $9(47.4)$ & \\
\hline No & 9 (52.9) & $10(52.6)$ & \\
\hline ECOG score, No. (\%) & & & 0.925 \\
\hline 0 & $2(11.8)$ & $2(10.5)$ & \\
\hline 1 & $10(58.8)$ & $12(63.2)$ & \\
\hline 2 & $5(29.4)$ & $5(26.3)$ & \\
\hline Histological type, No. (\%) & & & 0.676 \\
\hline Adenocarcinoma & $11(64.7)$ & $11(57.9)$ & \\
\hline Squamous carcinoma & $6(35.3)$ & $8(42.1)$ & \\
\hline TNM stage, No. (\%) & & & 0.955 \\
\hline IVA & $10(58.8)$ & $11(57.9)$ & \\
\hline IVB & $7(41.2)$ & $8(42.1)$ & \\
\hline \multicolumn{3}{|c|}{ Previous chemotherapy times, No. (\%) } & 0.865 \\
\hline 2 times & $10(58.8)$ & $10(52.6)$ & \\
\hline 3 times & 4 (23.6) & 6 (31.6) & \\
\hline 4 times & 3 (17.6) & $3(15.8)$ & \\
\hline
\end{tabular}

Comparison was determined by Student' $t$ test, Chi-square test and Wilcoxon rank sum test. DEB-BACE, drug-eluting beads bronchial arterial chemoembolization; SD, standard deviation; ECOG, Eastern Cooperative Oncology Group.

difference of ECOG score $(\mathrm{P}=0.925)$ or histological type $(\mathrm{P}=0.676)$ was found between the two groups. The detailed information of characteristics in NSCLC patients with refractory MPE were displayed in Table 1.

\section{MPE response in DEB-BACE group and chemotherapy group}

Patients in DEB-BACE group achieved better CR (82.4\% vs. $10.5 \%, \mathrm{P}<0.001)$, similar $\mathrm{PR}(17.6 \%$ vs. $42.1 \%, \mathrm{P}=0.112)$
Table 2 Comparison of MPE response between two groups

\begin{tabular}{|c|c|c|c|}
\hline Items & $\begin{array}{c}\text { DEB-BACE } \\
\text { group }(\mathrm{N}=17)\end{array}$ & $\begin{array}{c}\text { Chemotherapy } \\
\text { group }(\mathrm{N}=19)\end{array}$ & P value* \\
\hline CR, No. (\%) & $14(82.4)$ & $2(10.5)$ & $<0.001$ \\
\hline PR, No. (\%) & $3(17.6)$ & $8(42.1)$ & 0.112 \\
\hline Failure, No. (\%) & $0(0.0)$ & $9(47.4)$ & 0.001 \\
\hline Overall efficacy, No. (\%) & $17(100.0)$ & $10(52.6)$ & 0.001 \\
\hline \multicolumn{4}{|c|}{$\begin{array}{l}\text { *, Chi-square test. MPE, malignant pleural effusion; DEB-BACE, } \\
\text { drug-eluting beads bronchial arterial chemoembolization; CR, } \\
\text { complete remission; PR, partial remission; Overall efficacy = } \\
\text { CR+PR. }\end{array}$} \\
\hline
\end{tabular}

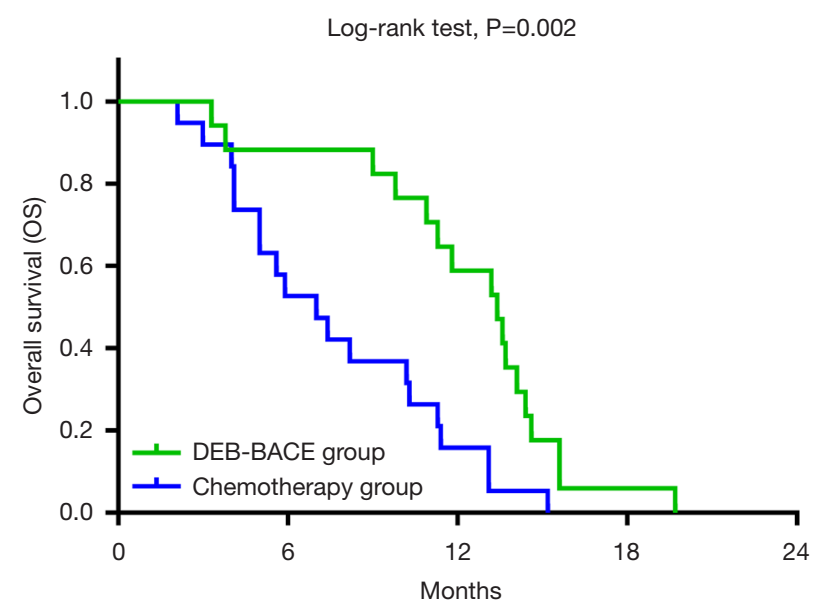

Figure 2 Comparison of OS between DEB-BACE group and chemotherapy group. DEB-BACE, drug-eluting beads bronchial arterial chemoembolization; CR, complete remission; PR, partial remission; MPE, malignant pleural effusion.

and less failure $(0.0 \%$ vs. $47.4 \%, \mathrm{P}=0.001)$ compared to patients in chemotherapy group (Table 2). As to the overall efficacy, it was increased in DEB-BACE group compared to chemotherapy group (100.0\% vs. $52.6 \%, \mathrm{P}=0.001)$ as well.

\section{OS in DEB-BACE group and chemotherapy group}

OS in DEB-BACE group and chemotherapy group were exhibited by K-M curves and compared by log-rank test, which showed that OS was prolonged in DEB-BACE group (median: 13.4 (95\% CI: 11.0-15.8 months) than that in chemotherapy group (median: 7.0 (95\% CI: 4.49.6 months) ( $\mathrm{P}=0.002)$ (Figure 2). To date, all 19 patients in chemotherapy group and all 17 patients in DEB-BACE group were dead. 
Table 3 Treatment efficacy in the DEB-BACE group

\begin{tabular}{|c|c|c|c|c|}
\hline Items & \multicolumn{3}{|c|}{ DEB-BACE treatment } & $\begin{array}{c}\text { Final } \\
\text { outcomes }\end{array}$ \\
\hline Treated patients, No. & 17 & 6 & 2 & 17 \\
\hline \multicolumn{5}{|l|}{ MPE response, No. (\%) } \\
\hline CR, No. (\%) & $11(64.7)$ & $2(33.3)$ & $1(50.0)$ & $14(82.4)$ \\
\hline Failure, No. (\%) & $1(5.9)$ & $0(0.0)$ & $0(0.0)$ & $0(0.0)$ \\
\hline Overall efficacy, No. (\%) & $16(94.1)$ & $6(100.0)$ & $2(100.0)$ & $17(100.0)$ \\
\hline \multicolumn{5}{|l|}{ ECOG score, No. (\%) } \\
\hline Improved & $11(64.7)$ & $1(16.7)$ & $0(0.0)$ & $12(70.6)$ \\
\hline
\end{tabular}

DEB-BACE, drug-eluting beads bronchial arterial chemoembolization; MPE, malignant pleural effusion; CR, complete remission; PR, partial remission; Overall efficacy $=\mathrm{CR}+\mathrm{PR}$; ECOG, Eastern Cooperative Oncology Group.

Table 4 Comparison of prognosis between CR and PR/failure patients who completed $1^{\text {st }}$ cycle of DEB-BACE treatment

\begin{tabular}{lcc}
\hline Items & CR patients $(n=11)$ & PR or failure patients $(n=6)$ \\
\hline ECOG score & & \\
Improved & $9(81.8)$ & $2(33.3)$ \\
Stable & $2(18.2)$ & $4(66.7)$ \\
MPE progression interval (months), median (95\% Cl) & $10.2(8.3-12.1)$ & $7.6(6.9-8.3)$ \\
Survival time (months), median (95\% Cl) & $13.6(13.1-14.1)$ & 0.015 \\
\hline
\end{tabular}

Comparison was determined by Chi-square test or log-rank test. CR, complete remission; PR, partial remission; ECOG, Eastern Cooperative Oncology Group; DEB-BACE, drug-eluting beads bronchial arterial chemoembolization; MPE, malignant pleural effusion.

\section{Detailed information of treatment outcomes by DEB- $B A C E$ plus intercostals arterial infusion chemotherapy}

After patients received $1^{\text {st }}$ cycle therapy $(\mathrm{n}=17), 11(64.7 \%)$, 5 (29.4\%), 1 (5.9\%) and $16(94.1 \%)$ patients had CR, PR, failure and overall efficacy respectively (Table 3); 11 $(64.7 \%)$ and $6(35.3 \%)$ patients presented with improved ECOG score and stable ECOG score respectively. After patients received $2^{\text {nd }}$ cycle therapy $(n=6), 2(33.3 \%), 4$ (66.7\%), 0 (0.0\%) and $6(100.0 \%)$ patients had CR, PR, failure and overall efficacy respectively; 1 (16.7\%) and $5(83.3 \%)$ patients showed improved ECOG score and stable ECOG score respectively. After patients received $3^{\text {rd }}$ cycle therapy $(\mathrm{n}=2), 1(50.0 \%), 1(50.0 \%), 0(0.0 \%)$ and $2(100.0 \%)$ patients had CR, PR, failure and overall efficacy respectively; $0(0.0 \%)$ and $2(100.0 \%)$ patients showed improved ECOG score and stable ECOG score respectively. Further analysis in patients who completed $1^{\text {st }}$ cycle of DEB-BACE treatment showed that: the improved ECOG score was more frequent $(\mathrm{P}=0.046)$, the median MPE progression interval was longer $(\mathrm{P}=0.015)$ while OS was similar $(\mathrm{P}=0.472)$ in $\mathrm{CR}$ patients compared to PR or failure patients (Table 4, Figure 3). Additionally, no association of age, gender, history of smoke, ECOG score or histological type with MPE response after $1^{\text {st }}$ cycle of DEB-BACE therapy was observed (all $\mathrm{P}<0.05$ ) (Table 5).

\section{Adverse events by DEB-BACE plus intercostals arterial infusion chemotherapy}

For the adverse events by DEB-BACE plus intercostals arterial infusion chemotherapy treatment, 4 (23.5\%), 5 (29.4\%), 2 (11.8\%), 2 (11.8\%), 1 (5.9\%) and $1(5.9 \%)$ 
A

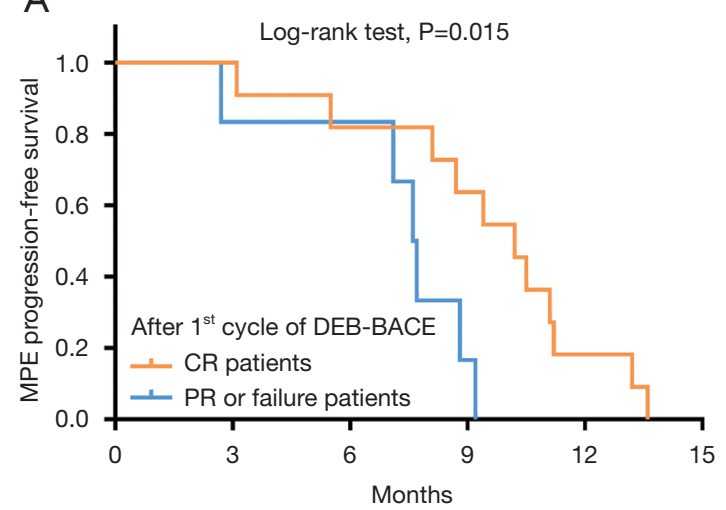

B

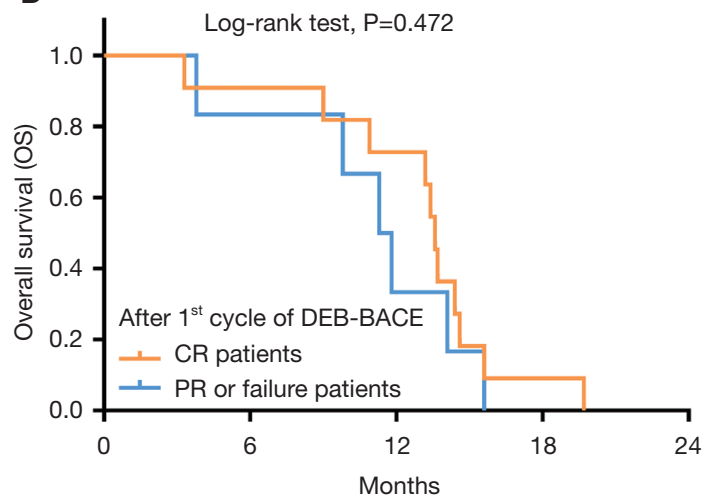

Figure 3 Comparison of MPE progression-free survival and OS between CR patients and PR/failure patients after $1^{\text {st }}$ cycle of DEB-BACE. MPE progression-free survival in CR and PR/failure patients after $1^{\text {st }}$ cycle of DEB-BACE (A); OS in CR and PR/failure patients after $1^{\text {st }}$ cycle of DEB-BACE (B). MPE, malignant pleural effusion; CR, complete remission; PR, partial remission; DEB-BACE, drug-eluting beads bronchial arterial chemoembolization.

Table 5 Association between characteristics and MPE response after $1^{\text {st }}$ cycle of DEB-BACE therapy in DEB-BACE group

\begin{tabular}{|c|c|c|c|c|}
\hline Items & No. & $\mathrm{CR}$ & PR or failure & $P$ value \\
\hline \multicolumn{4}{|l|}{ Age, No. (\%) } & 0.858 \\
\hline$<60$ years & 8 & $5(62.5)$ & $3(37.5)$ & \\
\hline$\geq 60$ years & 9 & $6(66.7)$ & $3(33.3)$ & \\
\hline \multicolumn{4}{|l|}{ Gender, No. (\%) } & 0.402 \\
\hline Male & 9 & $5(55.6)$ & $4(44.4)$ & \\
\hline Female & 8 & $6(75.0)$ & $2(25.0)$ & \\
\hline \multicolumn{4}{|c|}{ History of smoke, No. (\%) } & 0.858 \\
\hline Yes & 8 & $5(62.5)$ & $3(37.5)$ & \\
\hline No & 9 & $6(66.7)$ & $3(33.3)$ & \\
\hline \multicolumn{4}{|c|}{ ECOG score, No. (\%) } & 0.793 \\
\hline$<2$ & 12 & $8(66.7)$ & $4(33.3)$ & \\
\hline$\geq 2$ & 5 & $3(60.0)$ & $2(40.0)$ & \\
\hline \multicolumn{4}{|c|}{ Histological type, No. (\%) } & 0.349 \\
\hline Adenocarcinoma & 11 & $8(72.7)$ & $3(27.3)$ & \\
\hline $\begin{array}{l}\text { Squamous } \\
\text { carcinoma }\end{array}$ & 6 & $3(50.0)$ & $3(50.0)$ & \\
\hline
\end{tabular}

Comparison was determined by Chi-square test. MPE, malignant pleural effusion; DEB-BACE, drug-eluting beads bronchial arterial chemoembolization; $\mathrm{CR}$, complete remission; PR, partial remission; ECOG, Eastern Cooperative Oncology Group. patients suffered fever, chest distress/pain, gastrointestinal side effects, myelosuppression (Grade I), rash and hemoptysis respectively (Table 6). Notably, all these adverse events were mild, suggesting that DEB-BACE plus intercostals arterial infusion chemotherapy was tolerated in treating NSCLC patients with refractory MPE.

\section{Discussion}

From pathophysiological perspective, MPE arises from the malignant cells infiltrating to the pleural space, which further result in the accumulation of fluid in pleural space and contribute to the MPE formation, thus the effective treatments inhibiting tumor progression may help relieving MPE (1). The conventional chemotherapy relies on the ability of chemotherapeutic agents to control the accumulation of pleural fluids, either intravenously or intrapleurally administered, whereas the efficacy is limited and the systematic toxicity is increased (5,17-19). Then, the intra-arterial therapies are initiated with the idea of directly delivering the chemotherapeutic agents to the tumor through the tumor feeding arteries (7). The intercostals arterial infusion chemotherapy is a therapy that directly delivers the chemotherapy drugs to the tumor bed, which greatly increases the local drug concentration compared to conventional chemotherapy $(13,14)$. As to DEB-BACE, it provides embolization contributing to 
Table 6 Adverse events in DEB-BACE group

\begin{tabular}{ll}
\hline Adverse events & Patients (N=17) \\
\hline Fever, No. (\%) & $4(23.5)$ \\
Chest distress/pain, No. (\%) & $5(29.4)$ \\
Gastrointestinal side effects, No. (\%) & $2(11.8)$ \\
Myelosuppression (Grade I), No. (\%) & $2(11.8)$ \\
Rash, No. (\%) & $1(5.9)$ \\
Hemoptysis, No. (\%) & $1(5.9)$ \\
\hline
\end{tabular}

DEB-BACE, drug-eluting beads bronchial arterial chemoembolization.

tumor necrosis, meanwhile, microspheres are introduced for loading chemotherapeutic drugs, which are famous for the properties of facilitating sustained and controlled drug release (owing to the characteristics of its structure and the degradation of polymers) $(7,8)$. Thus, DEB-BACE ensures a permanent embolization to the bronchial artery and the sustained drug release, which further increases introtumoral drug concentration and decreases the systemic toxicity compared to conventional chemotherapy (7). In view of these particular characteristics of DEB-BACE and intercostals arterial infusion chemotherapy, both of them have the potential to bring clinical benefits for NSCLC patients with refractory MPE.

A few previous studies reveal the efficacy of conventional BACE in lung cancer patients. For instance, in one case report, a 91-year-old patient with advanced squamous cell lung cancer complicated with renal dysfunction is successfully treated by conventional BACE, with $87 \%$ of the tumor shadow in lung shrinkage (20). an interesting study shows that conventional BACE achieved elevated overall response rate (ORR) compared to bronchial arterial chemotherapeutic infusion $(71.4 \%$ vs. $42.4 \%)$ at 6 months after treatment in 33 patients with pulmonary metastases from hepatic cancer or other cancers (21). As to the limited evidence about DEB-BACE in lung cancers, one study displays that hemoptysis is improved by DEB-BACE in 10 advanced primary lung cancer patients (11); in a case report, a 56-year-old woman with refractory stage IV NSCLC who have been treated with repeated systemic chemotherapy shows multiple lung metastases, while after 6-month DEBBACE treatment, both the primary lesion in the right lower lobe and the invasion to the right main bronchus are improved (12). These studies highlight the good efficacy of DEB-BACE in relieving some manifestations of lung cancers, however, its efficacy in treating NSCLC patients with refractory MPE is unknown. In our study, 17 NSCLC patients with refractory MPE treated by DEBBACE plus the intercostals arterial infusion chemotherapy and 19 NSCLC patients with refractory MPE treated by conventional chemotherapy were included, and we compared the MPE response between the two groups. We observed that DEB-BACE plus the intercostals arterial infusion chemotherapy achieved better MPE response including increased CR, higher overall efficacy and less failure compared to the conventional chemotherapy. These results might be explained by the following reasons: (I) both the intercostals arterial infusion chemotherapy and DEBBACE were intra-arterial therapies, which directly delivered the drug to the tumor tissue or tumor bed, and dramatically increased the exposure of the tumor to anti-cancer agents and promoted cytotoxic effect to the tumor tissues compared to conventional chemotherapy, thereby DEB-BACE plus the intercostals arterial infusion chemotherapy was more effective in alleviating tumor progression and suppressing MPE formation, leading to increased CR, higher overall efficacy as well as less failure compared to conventional chemotherapy in NSCLC patients with refractory MPE (7); (II) DEB-BACE not only decreased the blood supply to tumor tissue, which facilitated tumor necrosis, but also slowed down the erosion of chemotherapeutic drugs in the blood stream and allowed the sustained and controlled drug release, resulting in a prolonged maintenance time of high-concentration chemotherapeutic drugs in tumor tissue compared to conventional chemotherapy, thus DEBBACE plus the intercostals arterial infusion chemotherapy showed better efficacy in inhibiting tumor progression and repressing the tumor cells infiltrate the pleural space, leading to the elevated MPE response compared to the conventional chemotherapy in treating MPE $(22,23)$.

According to previous studies, conventional BACE may provide survival benefit for lung cancer patients: a study enrolling 64 NSCLC patients shows that the median OS is 644 days in patients treated by conventional BACE plus radioactive iodine-125 seed implantation and 544 days in patients treated by conventional BACE alone, indicating the combination of conventional BACE and other treatments may further improved the prognosis of NSCLC patients (24). As for the survival of lung cancer patients after DEB-BACE treatment, the relative evidence is limited, only 
one study displays that 6 NSCLC patients underwent DEBBACE have the median OS of 16.5 months (7-23 months), which lacks a comparative control group (10). In this present study, we found that the median OS in DEB-BACE plus the intercostals arterial infusion chemotherapy treated patients was 13.4 (95\% CI: 11.0-15.8 months, which was remarkably increased than that in conventional chemotherapy treated patients (median 7.0 months; 95\% CI: 4.4-9.6 months), suggesting the superiority of DEB-BACE plus the intercostals arterial infusion chemotherapy compared to conventional chemotherapy for better prognosis in NSCLC patients with refractory MPE. This result might be on account of that (I) DEB-BACE primarily and directly delivered the drug to the tumor tissue or tumor bed, and dramatically increased the exposure of the tumor to anticancer agents and promoted cytotoxic effect to the tumor tissues, thereby DEB-BACE (plus the intercostals arterial infusion chemotherapy) was more effective in alleviating tumor progression and suppressing MPE formation; (II) MPE might be caused by the metastasis of pleura tumor, and the application of DEB-BACE might work on the lesion, and then suppresses MPE formation. Thus, DEBBACE and intercostals arterial infusion chemotherapy bring: the advantages of higher concentrations of chemotherapeutic agents load to the tumor tissue and the remarkably reduced systematic distribution of toxicity (compared to conventional chemotherapy) lead to better treatment response and attenuated tumor progression, therefore prolonged OS is observed in DEB-BACE plus intercostals arterial infusion chemotherapy treated patients than conventional chemotherapy treated patients (7).

Apart from the achievements in MPE response and survival, some unavoidable adverse events were also observed in NSCLC with refractory MPE patients after treated by DEB-BACE plus the intercostals arterial infusion chemotherapy. In detail, fever, chest distress/pain, gastrointestinal side effects, myelosuppression, rash and hemoptysis were observed, but the symptoms were relatively mild, indicating that DEB-BACE plus the intercostals arterial infusion chemotherapy was a tolerated therapy for NSCLC patients with refractory MPE.

Some limitations existed in our study: (I) this was a single-center study, which might cause selection bias, and multi-center study was needed to validate these findings; (II) the sample size was small, which might result in a weak statistical power; however, the application of DEB-BACE plus intercostals arterial infusion chemotherapy was a novel treatment strategy for NSCLC patients with refractory
MPE, thus the number of patients receiving this therapy was limited, and further validations in studies with larger sample size was required; (III) In this study, 19 NSCLC patients with refractory MPE treated by conventional chemotherapy were reviewed retrospectively, and the data of these patients were collected from medical records. Hence, data was not corrected about post-treatment ECOG scores of the control group for comparison with the baseline scores and with the post treatment scores of the intervention group. Further study was needed. (IV) the control group was a cohort of conventional chemotherapy, while a further study with control group of conventional BACE or intercostals arterial infusion chemotherapy would better reflect the efficacy of DEB-BACE plus intercostals arterial infusion chemotherapy; $(\mathrm{V})$ this present study was non-randomized, thus confounding factors might existed.

In conclusion, DEB-BACE plus intercostals arterial infusion chemotherapy is an effective and tolerated therapy for NSCLC patients with refractory MPE.

\section{Acknowledgments}

Funding: This study was supported by National Natural Science Foundation of China (No. 81901935), Military Youth Development Program (No. 17QNP034), Logistic Support Department of CMC Health Care Project (No. 21BJZ42) and National Natural Science Foundation of Beijing (No.7212104).

\section{Footnote}

Data Sharing Statement: Available at http://dx.doi. org/10.21037/jtd-20-1603

Conflicts of Interest: All authors have completed the ICMJE uniform disclosure form (available at http://dx.doi. org/10.21037/jtd-20-1603). The authors have no conflicts of interest to declare.

Ethical Statement: The authors are accountable for all aspects of the work in ensuring that questions related to the accuracy or integrity of any part of the work are appropriately investigated and resolved. The study was conducted in accordance with the Declaration of Helsinki (as revised in 2013). All patients who were prospectively recruited in our study provided written informed consent themselves, while for the patients in control cohort who were retrospectively enrolled but had died, so we had to 
obtain the written consents from their families. Additionally, this study was registered on Chinese Clinical Trial Registry (http://www.chictr.org.cn), with the registry number of ChiCTR2000033063.

Open Access Statement: This is an Open Access article distributed in accordance with the Creative Commons Attribution-NonCommercial-NoDerivs 4.0 International License (CC BY-NC-ND 4.0), which permits the noncommercial replication and distribution of the article with the strict proviso that no changes or edits are made and the original work is properly cited (including links to both the formal publication through the relevant DOI and the license). See: https://creativecommons.org/licenses/by-nc-nd/4.0/.

\section{References}

1. Skok K, Hladnik G, Grm A, Crnjac A. Malignant Pleural Effusion and Its Current Management: A Review. Medicina (Kaunas) 2019;55:490.

2. Murthy P, Ekeke CN, Russell KL, et al. Making cold malignant pleural effusions hot: driving novel immunotherapies. Oncoimmunology 2019;8:e1554969.

3. McCracken DJ, Porcel JM, Rahman NM. Malignant Pleural Effusions: Management Options. Semin Respir Crit Care Med 2018;39:704-12.

4. Gompelmann D, Eberhardt R, Herth FJ. Advanced malignant lung disease: what the specialist can offer. Respiration 2011;82:111-23.

5. Epelbaum O, Rahman NM. Contemporary approach to the patient with malignant pleural effusion complicating lung cancer. Ann Transl Med 2019;7:352.

6. Desai NR, Lee HJ. Diagnosis and management of malignant pleural effusions: state of the art in 2017. J Thorac Dis 2017;9:S1111-22.

7. Savic LJ, Chapiro J, Geschwind JH. Intra-arterial embolotherapy for intrahepatic cholangiocarcinoma: update and future prospects. Hepatobiliary Surg Nutr 2017;6:7-21.

8. Wáng YX, De Baere T, Idee JM, et al. Transcatheter embolization therapy in liver cancer: an update of clinical evidences. Chin J Cancer Res 2015;27:96-121.

9. Seki A, Hori S, Sueyoshi S, et al. Transcatheter arterial embolization with spherical embolic agent for pulmonary metastases from renal cell carcinoma. Cardiovasc Intervent Radiol 2013;36:1527-35.

10. Bie Z, Li Y, Li B, et al. The efficacy of drug-eluting beads bronchial arterial chemoembolization loaded with gemcitabine for treatment of non-small cell lung cancer. Thorac Cancer 2019;10:1770-78.

11. Seki A, Shimono C. Transarterial chemoembolization for management of hemoptysis: initial experience in advanced primary lung cancer patients. Jpn J Radiol 2017;35:495-504.

12. Kennoki N, Hori S, Yuki T, et al. Trans-Arterial Chemoembolization Therapy for Refractory Advanced Non-Small Cell Lung Cancer with Spherical Embolic Material--A Single Case Report. Gan To Kagaku Ryoho 2015;42:1827-9.

13. Vogl TJ, Shafinaderi M, Zangos S, et al. Regional chemotherapy of the lung: transpulmonary chemoembolization in malignant lung tumors. Semin Intervent Radiol 2013;30:176-84.

14. Kahn PC, Paul RE, Rheinlander HF. Selective bronchial arteriography and intra-arterial chemotherapy in carcinoma of the lung. J Thorac Cardiovasc Surg 1965;50:640-5.

15. Millar JW, Hunter AM, Horne NW. Intrapleural immunotherapy with Corynebacterium parvum in recurrent malignant pleural effusions. Thorax 1980;35:856-8.

16. Qi N, Li F, Li X, et al. Combination use of paclitaxel and avastin enhances treatment effect for the NSCLC patients with malignant pleural effusion. Medicine (Baltimore) 2016;95:e5392.

17. Tao H, Meng Q, Li M, et al. Outcomes of bevacizumab combined with chemotherapy in lung adenocarcinomainduced malignant pleural effusion. Thorac Cancer 2018;9:298-304.

18. Fujita A, Takabatake H, Tagaki S, et al. Combination chemotherapy in patients with malignant pleural effusions from non-small cell lung cancer: cisplatin, ifosfamide, and irinotecan with recombinant human granulocyte colonystimulating factor support. Chest 2001;119:340-3.

19. Figlin R, Mendoza E, Piantadosi S, et al. Intrapleural chemotherapy without pleurodesis for malignant pleural effusions. LCSG Trial 861. Chest 1994;106:363S-66S.

20. Kunisada K, Ishikawa H, Takafuji J, et al. A case of a 91-year-old patient with advanced squamous cell lung cancer complicated with renal dysfunction successfully treated with trans-arterial chemo-embolization. Gan To Kagaku Ryoho 2013;40:917-9.

21. Jiang GM, Zhao JW, Chen YX, et al. Blood supply of pulmonary metastases and its clinical significance. Ai Zheng 2006;25:885-7. 
22. Hong K, Khwaja A, Liapi E, et al. New intra-arterial drug delivery system for the treatment of liver cancer: preclinical assessment in a rabbit model of liver cancer. Clin Cancer Res 2006;12:2563-7.

23. Varela M, Real MI, Burrel M, et al. Chemoembolization of hepatocellular carcinoma with drug eluting beads:

Cite this article as: Liu $\mathrm{X}$, Lin $\mathrm{H}$, Wang Q, Mu M, Pan P, Tian F, Zhang R, Zhao W, Bao P. Drug-eluting beads bronchial arterial chemoembolization plus intercostals arterial infusion chemotherapy is effective and well-tolerated in treating nonsmall cell lung cancer patients with refractory malignant pleural effusion. J Thorac Dis 2021;13(4):2339-2350. doi: 10.21037/jtd-201603 efficacy and doxorubicin pharmacokinetics. J Hepatol 2007;46:474-81.

24. Chen Y, Li Y, Jia Y, et al. Bronchial artery chemoembolization combined with radioactive iodine-125 seed implantation in the treatment of advanced nonsmall cell lung cancer. J Cancer Res Ther 2017;13:636-41. 